\title{
Evaporative cooling in microfluidic channels
}

\author{
George Maltezos, Aditya Rajagopal, and Axel Scherer ${ }^{\mathrm{a})}$ \\ Caltech, Pasadena, California 91125
}

(Received 29 March 2006; accepted 19 June 2006; published online 18 August 2006)

\begin{abstract}
Evaporative cooling is an effective and energy efficient way to rapidly remove heat from a system. Specifically, evaporative cooling in microfluidic channels can provide a cost-effective solution for the cooling of electronic devices and chemical reactors. Here we present microfluidic devices fabricated by using soft-lithography techniques to form simple fluidic junctions between channels carrying refrigerant and channels carrying $\mathrm{N}_{2}$ gas. The effects of channel geometry and delivery pressure on the performance of refrigeration through vaporization of acetone, isopropyl alcohol, and ethyl ether were characterized. By varying gas inlet pressures, refrigerants, and angles of the microfluidic junctions, optimal cooling conditions were found. Refrigeration rates in excess of $40{ }^{\circ} \mathrm{C} / \mathrm{s}$ were measured, and long lasting subzero cooling in the junction could be observed. (C) 2006 American Institute of Physics. [DOI: 10.1063/1.2234318]
\end{abstract}

Miniaturization of components has been the defining trend in the world of electronics and optics for the past 50 years. Moore's law predicts a doubling of transistor density in approximately every 18 months, ${ }^{1}$ and transistor resolution is now well below $100 \mathrm{~nm}^{2}$ With this rapid increase in transistor density, the fundamental problem of heat dissipation places ultimate limitations on processing power and device speed. Attention to energy consumption and heat dissipation is of paramount importance ${ }^{3}$ when designing electronic and optical architectures. So far, heat dissipation has been addressed in a variety of ways, from "sleep" transistors ${ }^{4}$ to on-chip microrefrigeration. ${ }^{5-7}$ Apart from the electronic applications of refrigeration, there are many other needs for miniaturized refrigerators, including optical and microwave detector cooling, polymerase chain reactor cycling, and thermal stabilization of high power telecommunication lasers. Temperature control has also become extremely important in ensuring the precise control of thermally sensitive reactions within the framework of microfabricated chemical "laboratories" ${ }^{\text {-10 }}$ in which subnanoliter volumes of reagents are reacted on microfluidic chips. Here we will describe the integration of evaporative cooling through microfluidic channels to address the needs of these applications.

We present a unique solution to the problem of heat dissipation: localized cooling through evaporation of volatile materials within microfluidic channels. It is well known that refrigeration can be achieved through the endothermic mixing of compressed gas with an evaporating liquid. ${ }^{11}$ To show that this also applies in microfluidic geometries, small channels were fabricated to carry out the refrigeration, and connected to form simple $Y$ junctions with two input channels (see Fig. 1)—one for the refrigerant and the other for the gas. Evaporation occurs throughout the outlet channel. In our experiments, each channel has a length of $6.5 \mathrm{~mm}$ and a diameter of $0.650 \mathrm{~mm}$. The angle of the $Y$ junction was varied between $10^{\circ}$ and $180^{\circ}$, and the cooling effect was characterized as a function of angle, inlet gas pressure $\left(\mathrm{N}_{2}\right)$, and type of refrigerant. Furthermore, we characterized the refrigera-

${ }^{\text {a) }}$ Author to whom correspondence should be addressed; electronic mail: etcher@caltech.edu tion using ethyl ether, acetone, ethyl alcohol, and isopropyl alcohol. The fluidic channels were initially designed on a computer using SOLIDWORKS, a three-dimensional modeling tool. These models were then converted to a usable file format using SolidScape's MODELWORKS software. Wax molds of the fluidic channels were created using a SolidScape T66 wax printer. The wax molds were then chemically cured (to remove unwanted build wax) with Petroferm Bioact VS-0 precision cleaner, and thermally cured by overnight heating at $37^{\circ} \mathrm{C}$.

Sylgard Dow-Corning 184 polydimethalsiloxane (PDMS) elastomer was mixed in a Keyence hybrid mixer HM501 to fabricate the fluidic channels. The PDMS was then cured in a two step process. First, a $0.3 \mathrm{~mm}$ thick initial layer of elastomer was degassed in a vacuum chamber for $10 \mathrm{~min}$ and thermally cured at $80{ }^{\circ} \mathrm{C}$ for $8 \mathrm{~min}$. A second, thin layer of PDMS was then poured on top of the first layer. The wax junction mold was then placed on the second layer of PDMS. Finally, a $3 \mathrm{~mm}$ thick layer of PDMS was poured on top of the wax mold. The setup was finally pumped down in a vacuum chamber for $25 \mathrm{~min}$ and then heated at $54{ }^{\circ} \mathrm{C}$ for $4 \mathrm{~h}$ to degas the elastomer.

The PDMS blocks were then cropped and dewaxed (by heating to $90{ }^{\circ} \mathrm{C}$ for $15 \mathrm{~min}$ ). Acetone was rinsed through the fluidic channels to remove residual build wax. The fluidic chips were then attached to a refrigerant and $\mathrm{N}_{2}$ gas inlets. An Omega Precision fine wire, $K$-type thermocouple was inserted into the outlet of the fluidic channel. The thermocouple had a diameter of $0.125 \mathrm{~mm}$ - small enough not to interfere with the outlet of refrigerant and gas. The thermocouple was attached to an Omega iSeries i/32 temperature controller that logged the temperature in the junction at a rate

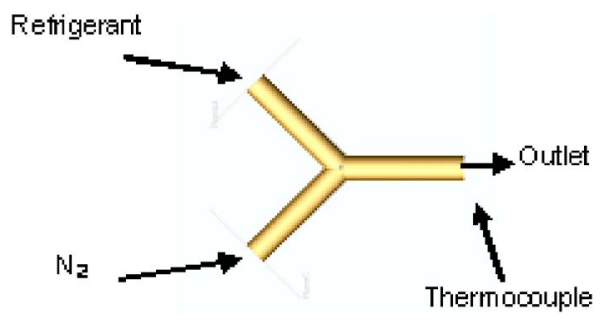

FIG. 1. (Color online) Fluidic chip layout. 


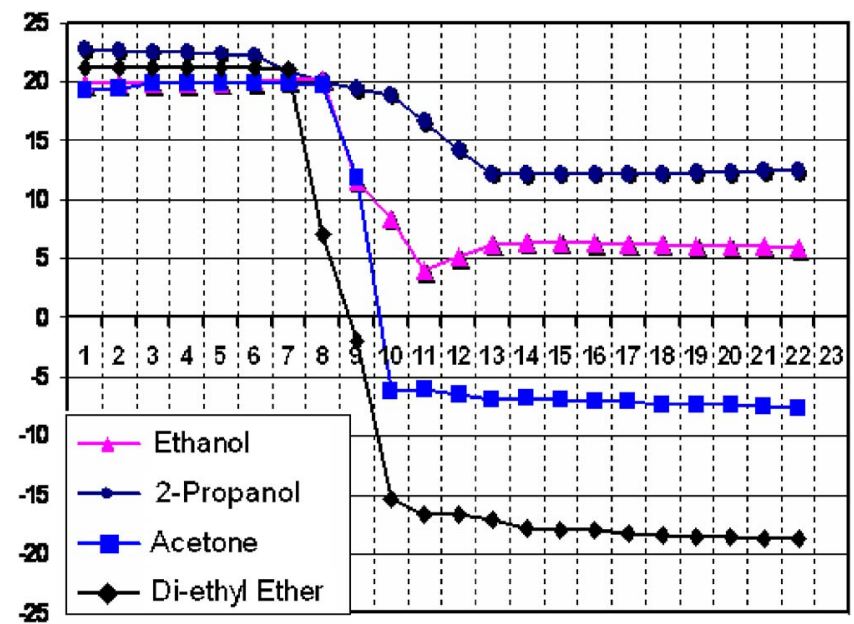

FIG. 2. (Color online) Temperature drop vs time for four refrigerants.

of approximately three times a second. Temperature measurements were made using this controller which was interfaced to a computer via serial port and Microsoft HyperTerminal. The inlet pressures of the refrigerant and the gas were monitored by TIF Instruments digital pressure meters.

Preliminary characterization of the channels indicates that the lowest refrigeration temperatures were achieved by evaporating ethyl ether. We believe that this is due to the very low specific heat and boiling point of ethyl etherenabling rapid vaporization. Refrigeration rates recorded for the vaporization of ethyl ether (ethyl ether pressure was 1.2 psi and $\mathrm{N}_{2}$ inlet pressures were in excess of $20 \mathrm{psi}$ ) were approximately $40{ }^{\circ} \mathrm{C} / \mathrm{s}$. The evaporation of ethyl ether also enabled temperatures as low as $-20^{\circ} \mathrm{C}$ to be sustained for several minutes (prolonged cooling effects in excess of 15 min were recorded) (see Fig. 2).

The rates of temperature decrease in the mixing chambers were also characterized with respect to the gas inlet pressures. We incremented gas inlet pressures from 0 to 36 psi in steps of 3 psi, and found that there was a positive relationship between the gas inlet pressure and the refrigeration rate. However, we noted that inlet pressures in excess of 21 psi did not significantly increase the cooling effect further (see Fig. 3). We suspect that such increases in the gas inlet pressure create back pressure within the inlet

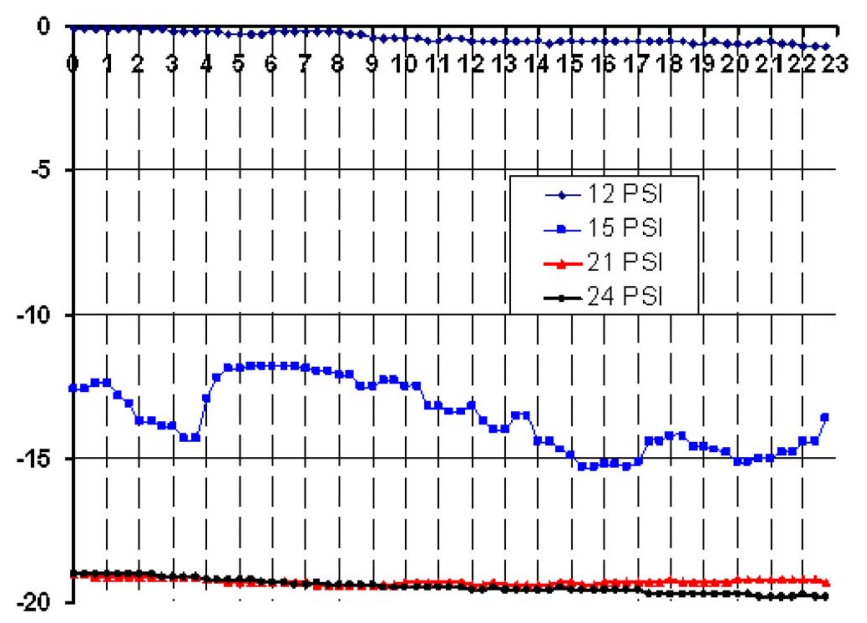

FIG. 3. (Color online) Graph of minimal attainable temperature (centigrade) vs time (seconds) for two channels impinging at $10^{\circ}$. vs time (seconds) for two channels impinging at $10^{\circ}$. conservation would employ a selective, organic membrane at
Downloaded 08 Sep 2006 to 131.215 .225 .158 . Redistribution subject to AIP license or copyright, see http://apl.aip.org/apl/copyright.jsp

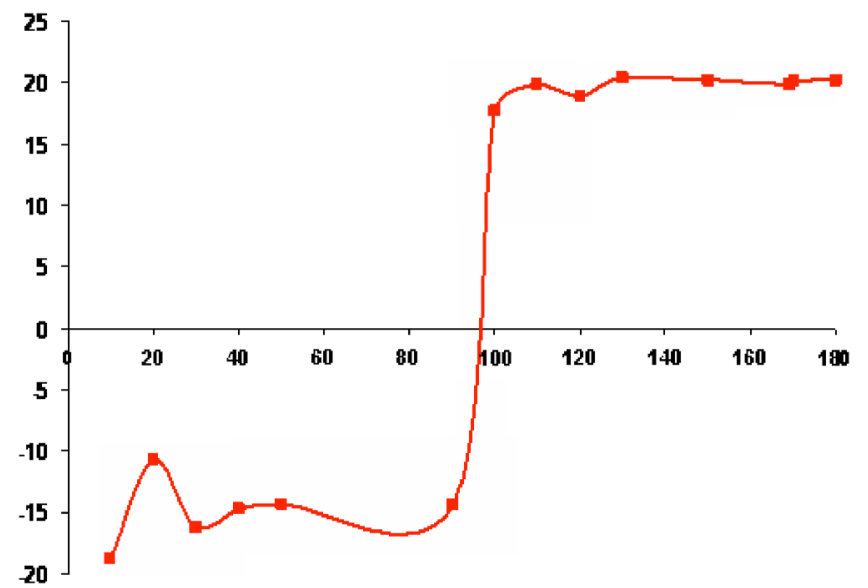

FIG. 4. (Color online) Minimum attainable temperature (centigrade) vs angle of junction.

channels, and a corresponding disruption in the flow of the refrigerant. Such back pressure contributes to erratic temperature fluctuations and a minimized cooling effect.

We also characterized $Y$-junction geometries with respect to angle between the channels. We found that the slowest refrigeration occurs when the angle between the channels forming the $Y$ branch is large $\left(180^{\circ}\right)$, and the refrigerant supply and nitrogen evaporation gas sources point at each other. Conversely, the most efficient cooling was measured when the angle between these two supply channels was $10^{\circ}$ $Y$ junction. We think that this is due to the fact that in smaller angles, flow congestion at the junction can be minimized. Smaller angles allow the vaporized refrigerant and heat to leave the system faster. Furthermore, larger angles exhibit significant back pressure problems at pressures in excess of 15 psi (see Fig. 4). Back pressure prevents proper refrigerant flow, thereby dampening the cooling effect. This was most clearly demonstrated in a test (gas inlet was 25 psi and ethyle ether inlet was $1.2 \mathrm{psi}$ ) for the $180^{\circ}$ junction, where little or no cooling was observed.

Localized evaporative cooling in fluidic channels provides an elegant and low-cost solution to the problem of cooling electronics, optics, and chemical reactions. We optimizing the geometry of a $Y$-junction and the gas/evaporant ratios $^{11}$ for this purpose. We found that the optimal refrigeration took place in a $10^{\circ} \mathrm{Y}$ junction when using ethyl ether as an evaporant. Maximum temperature drops were measured when gas inlet pressures were between 21 and 36 psi. Gas inlet pressures above 36 psi resulted in dampened cooling effects. We also investigated the increase of the size of the outlet channel (outlet channel had a diameter of $2 \mathrm{~mm}$ ) in hopes of promoting the expansion associated with volatilization of the refrigerant. However, we found that widening the outlet channel did not result in faster refrigeration.

A copper heat sink was also embedded into a fluidic chip. In this chip, it was observed that the cooling effect in the $Y$-junction channel results in a cooling effect in a second channel. The copper heat sink acts as a thermal bridge between the two channels. With this model, it is possible to demonstrate the cooling of an external heat source. This experiment confirms that it is possible to apply the evaporative coolers for electronic and optical cooling purposes. Ultimately, we hope to develop self-sustaining, closed systems in which refrigerant is conserved. One possibility for refrigerant 
the output channel. Furthermore, in order to provide an applicable solution for the cooling of electronics, we plan on integrating microfluidic cooling systems into silicon.

The authors would like to thank Alvaro Gomez for helping them realize the initial setup of the experiment, and Michael David Henry for thought provoking discussions. This project was supported by generous grants from the National Science Foundation Center for Nano-chemicalelectrical Mechanical Manufacturing Systems (NSFCEMMS) under DMI-0328162 and from the BOEING company under the Multifunctional NanoSystem Technologies program. Two of the authors (G. M. and A. R.) Contributed equally to this research.

${ }^{1}$ V. Zhirnov, R. Cavin, J. Hutchby, and G. Bourianoff, Proc. IEEE 91
(2003).

${ }^{2}$ Intel Corporation; http://www.intel.com/technology/silicon/mooreslaw/ ${ }^{3}$ HP Development Company; http://www.hpl.hp.com/news/2003/oct_dec/ energy_talk.html

${ }^{4}$ J. Park, V. Moony, and P. Pfeiffenberger, Manuscript: http:// codesign.ece.gatech.edu/publications/jcpark/paper/patmos_2004.pdf

${ }^{5}$ A. Shakouri and Y. Zhang, IEEE Trans. Compon. Packag. Technol. 28 (2005).

${ }^{6}$ S. Borkar, IEEE Micro. 19, 23-29 (1999).

${ }^{7}$ G. Guijt, A. Dodge, G. van Dedem, N. Rooij, and E. Verpoorte, Lab Chip 3, 1-4 (2003).

${ }^{8}$ E. Hasselbrink, Jr., T. Shepodd, and J. Rehm, Anal. Chem. 74, 4913 (2002).

${ }^{9}$ J. Xie, Y. Miao, J. Shih, Q. He, J. Liu, Y. Tai, and T. Lee, Anal. Chem. 76, 3756 (2004).

${ }^{10}$ A. Pattekar and M. Kothare, J. Micromech. Microeng. 13, 337 (2003). ${ }^{11}$ CRC Handbook of Chemistry and Physics (76th edition) (CRC Press, New York, 1999), pp. 6-120. 